\title{
Innovación para el envejecimiento activo en la unión europea. Análisis del programa ambient assisted living joint programme (aal) en el periodo 2008-2015
}

\author{
Sacramento Pinazo-Hernandis ${ }^{1}$, Rakel Poveda Puente ${ }^{2}$ \\ Recibido: 23-09-2015 - Aceptado: 02-12-2015
}

\begin{abstract}
Resumen
Los productos y servicios centrados en las personas, diseñados y seleccionados teniendo en cuenta las necesidades, las características de los usuarios y sus capacidades facilitan la vida independiente a todas ellas con o sin limitaciones funcionales. El programa Ambient Assisted Living Joint Programme $(\mathrm{AAL})$, es un ejemplo de cómo se pueden desarrollar proyectos basados en las TIC que permitan al adulto permanecer en su hogar el mayor tiempo posible de forma autónoma e independiente. Tomando como referente el Paradigma del envejecimiento activo y una vez conocidos y analizados los diferentes proyectos del Programa AAL (2008-2015) de desarrollo de tecnologías de ayuda y apoyo a las personas mayores, los tipos de proyectos financiados y las áreas de desarrollo, los objetivos que se plantea en esta investigación fueron: Analizar los proyectos evaluados positivamente y realizados en los últimos 7 años, y reflexionar sobre el futuro de las tecnologías en la promoción del envejecimiento activo. Metodología. Tras una lectura en profundidad de los propósitos de cada convocatoria y mediante una rejilla de análisis, dos expertos entrenados revisaron el grado de cercanía entre los objetivos de la convocatoria AAL y el Paradigma de envejecimiento activo. Resultados. Los proyectos revisados se han dirigido a la promoción de la salud y la actividad autónoma y a la monitorización de la salud, en áreas como la inclusión social, prevención, supervisión, detección, y apoyo. También ha habido proyectos dirigidos al fomento de la participación, algunos centrados en la seguridad, y otros que han buscado trabajar todas las áreas conjuntamente. Conclusiones. El artículo muestra la variedad de proyectos AAL centrados en tecnologías de ayuda y de apoyo a la vida independiente y al envejecimiento activo. Se posee la convicción de la necesidad de que los profesionales conozcan los proyectos que se están desarrollando en la Unión Europea y la importancia de conectar con los profesionales y con las personas mayores para la investigación e innovación en este ámbito.
\end{abstract}

Palabras clave: Envejecimiento activo; TICs; vida independiente; diseño centrado en la persona.

\footnotetext{
${ }^{1}$ Doctora en Psicología. Profesora titular de la Universidad de Valencia. Facultad de Psicología, España; sacramento.pinazo@uv.es ${ }^{2}$ Magíster en intervención social. Investigadora del Instituto de Biomecánica de Valencia. Profesora asociada del Departamento Trabajo Social y Servicios Sociales. Universidad de Valencia, España; rakel.poveda@ibv.upv.es
} 
Sacramento Pinazo-H., Rakel Poveda P., - Innovación para el envejecimiento activo en la unión europea

\title{
Innovation for active aging in the european union. Analysis of the ambient assisted living joint programme (aal) program for the period 2008-2015
}

\begin{abstract}
Products and services focused on people, designed and selected taking into account the needs, characteristics of users and their capabilities can facilitate independent living to all people with or without functional limitations. The Ambient Assisted Living Joint Programme (AAL) is an example about how the development of ICT can be focused to enable older people to remain at home as long as possible autonomously and independently. Based on the Paradigm of Active Ageing we have known and analyzed the different projects in the AAL Programme (2008-2015) about the development of assistive technologies and support for elderly people, depending on the types of projects funded and the development area, Our goals were: to analyse possitive evaluated projects and reflect about the future of ICT to promote active ageing. Method. Beginning with a in-depht reading about call's goals and through a grilled analysis, two trained experts reviewed the levels of proximity between AAL calls and Active Ageing model. Results. The proyects reviewed were aimed at health promotion and autonomous activity, and health monitoring in areas such as social inclusion, prevention, monitoring, detection, and supervision. There were also projects aimed at promoting participation, others focused on safety, and others taking into account all areas as a whole. Conclusions. This article shows the diversity of AAL projects focused on ICTs to promote independent life and active ageing. We are convinced that professionals need to know the amount of projects developped around European Union and the importance of connecting with professionals and elderly people for an increase of research and innovation involved in this area.
\end{abstract}

Keywords: Active Ageing; ICT; independent living; person-oriented design.

\section{Introducción}

El envejecimiento poblacional está generando nuevas demandas en las estructuras sociales y sanitarias dirigidas a las personas mayores, en general, y a aquellas personas en situación de dependencia, en particular. En 2010, 524 millones de personas tenían 65 años (8\% población mundial) (OMS, 2010). Las previsiones indican que desde el año 2015 hasta el 2050 la población mundial mayor de 60 años se incrementará de 900 millones a 2.000 millones de personas (OMS, 2015). Dentro de veinte años la edad media de los ciudadanos en países como Alemania, Italia o España rozará los 50, y será aún mayor en el caso de Japón. En la Unión Europea, la esperanza de vida una vez superados los 65 años seguirá aumentando un año cada ocho (Índice Global de Envejecimiento, 2013). El proceso de envejecimiento conlleva modificaciones que influyen en la capacidad funcional, y por consiguiente en la realización actividades de la vida diaria (AVD). La Comisión Europea (2003) indico que entre $6,8-14,1 \%$ de la población de adultos mayores (10,2-21,2 millones de personas) tienen una ligera a moderada limitación física que dificulta la realización de las AVD de forma autónoma. La probabilidad de necesitar atención aumenta con edad. Según el estudio de Colombo (2011) menos de 1\% de los menores de 65 años utiliza cuidados de larga duración, mientras que el 30\% de las mujeres de más de 80 años de edad los utiliza; el cuidado ofrecido por los familiares es mucho más frecuente en España (15 a 16\% de la población presta cuidado no profesional) que en otros países de la Unión Europea. La persona que fundamentalmente cuida de las personas mayores con dependencia es su cónyuge, seguida de su hija con una edad media de 60 años (IMSERSO, 2012).

El envejecimiento no es solo un fenómeno de los países desarrollados. El aumento de la longevidad es un motivo de celebración, pero a la vez el envejecimiento representa un desafío si se quiere maximizar el potencial de la población 
BÚSQUEDA - Julio / Diciembre de 2015 - No. 15 (38 - 50)

mayor, pues las personas mayores también contribuyen a sus familias y a sus comunidades (Índice Global de Envejecimiento, 2013). La Comisión Europea a través de diferentes informes ha desarrollado en los últimos veinte años una línea de pensamiento que combina la incertidumbre del coste del envejecimiento con la oportunidad del valor añadido del mismo. Ya en 1999 la Comisión Europea publicó el informe Hacia una Europa para todas las edades, en donde decía que era necesario innovar en mejoras en la calidad sanitaria y de los servicios para las personas mayores que evitasen la exclusión social. Merece la pena recordar también algunos otros documentos como el de 2006, El futuro demográfico: transformar un reto en una oportunidad, o el de 2007, Promover la solidaridad entre generaciones, que plantean estas mismas inquietudes.

Pensando en la atención a las personas mayores en el próximos decenio, los políticos, gestores, profesionales e investigadores se deberán enfrentar a diferentes cuestiones relacionadas con la atención integral a las personas mayores, como son: el mantenimiento de la salud conforme las personas avanzan en edad; la mejora de los entornos que faciliten la realización de las actividades de la vida diaria de la manera más autónoma posible, y los cuidados de larga duración. La multifactorialidad de estos retos supone un abordaje desde distintas disciplinas con una visión social, económica, legislativa, biomédica, ética y tecnológica con participación activa de las personas mayores y sus familiares, como principales actores y cuyos resultados sean aceptados y usados por la ciudadanía que, tal y como indica Echevarría (2008) son los criterios básicos del éxito de la innovación social.

La innovación social debe tener en cuenta a las personas a lo largo de sus vidas, y concretamente prestar atención a sus necesidades durante el proceso de envejecer. Los cambios a lo largo del ciclo vital y los distintos modos de afrontarlos hacen que nos encontremos con un grupo de personas mayores muy variado. Variables como el sexo, edad, estado civil, nivel de estudios, nivel de ingresos, percepción de salud, funcionalidad, apoyo social, o formas de convivencia marcan la heterogeneidad de este grupo de población. Y muchas de las diferencias están relacionadas con el entorno en el que viven (siguiendo el Informe del la II Asamblea Mundial del Envejecimiento, Madrid International Plan of Action on Ageing, MIPAA): "que los entornos físicos tengan en cuenta a las personas mayores establece la diferencia entre independencia y dependencia".

El International Longevity Center de Brasil (ILCB), think-tank independiente, ubicado en Rio de Janeiro, basado en investigaciones internacionales tiene como misión difundir ideas y políticas que guíen a la población que envejece, y siempre con el punto de mira puesto en el envejecimiento activo, redactó en 2015 el informe Envejecimiento activo: un marco político para dar respuesta a la revolución de la longevidad, en la cual se indica que el envejecimiento converge con otras tendencias globales que afectan a los individuos a lo largo de la vida, como la revolución tecnológica, y dentro de esta, el desarrollo de sistemas para la gestión de la salud, el autocuidado, la atención domiciliaria, el fomento de la vida activa que permiten y mantienen la independencia y la participación. Tal y como indica el citado informe "El envejecimiento es un potente motor de la innovación tecnológica y a su vez está cambiando lo que significa ser una persona mayor. Sin embargo, el acceso a los beneficios de la tecnología es profundamente desigual; la disponibilidad y la asequibilidad son los principales obstáculos para el beneficio universal de la innovación tecnológica".

Años atrás, también el proyecto Envejecimiento Saludable -financiado en el marco del programa de Salud Pública de la Unión Europea 20032008 (European Commission, 2007), subrayaba que los sistemas para afrontar el envejecimiento debían incorporar políticas de promoción de la salud a lo largo del ciclo vital, con un claro objetivo: disminuir el impacto sobre la economía, la asistencia, el desarrollo social para lograr el bienestar de las sociedades europeas. 
Sacramento Pinazo-H., Rakel Poveda P., - Innovación para el envejecimiento activo en la unión europea

Por su parte, la Estrategia Horizonte 2020 -cuyo fin es estimular la investigación e innovación que permitan alcanzar los objetivos políticos de la Unión, entre otros temas-, se plantea los siguientes retos relacionados con el envejecimiento y las tecnologías hacia los cuales deberán orientarse las investigaciones: salud, cambio demográfico y bienestar, y hacer de las ciudades europeas sociedades inclusivas, innovadoras y reflexivas. E indicando que todas las actividades aplicarán un enfoque basado en dar respuesta a los retos que afronta la sociedad, incluyendo investigación básica o aplicada, transferencia de tecnología o innovación, y también la innovación no tecnológica y la organizativa, así como innovación en el sector público.

Ante este panorama, ¿qué ofrece la tecnología a las personas mayores? Según el Informe del CEAPAT, Tecnología y personas mayores (2015), si pensamos en las personas mayores como usuarios de las tecnologías, estas proporcionan acceso a la comunicación, la formación o el ocio, además de que en sí mismas son motivadoras y divertidas; pero si pensamos en las personas mayores como perceptores de servicios apoyados en las tecnologías de la información y la comunicación (TIC), les permiten prolongar su vida en el entorno natural con mayor seguridad, con un contacto personal con las personas del Centro social o sanitario, lo que disminuye la sensación de aislamiento, sobre todo en el caso de las personas mayores que viven solas (CEAPAT, 2015).

Encontrar un equilibrio entre la tecnología y las necesidades de las personas mayores es básico para el desarrollo de estrategias adecuadas. Siguiendo a Poveda, Belda, Barberà, Cort, Prat, Matey, Soler, y Dejoz, 2005: 155): "La realización de las actividades de la vida diaria, tanto las básicas, las instrumentales, como las avanzadas, implica una interacción con el entorno más inmediato y con los elementos que lo componen. Lamentablemente, los entornos son diseñados para usuarios-tipo que no se corresponden con la realidad de muchas de las personas mayores, y menos aun cuando estas personas tienen algún tipo de alteración funcional. Así, un espacio diseñado para una persona joven (generalmente hombre y sin ninguna limitación funcional) resultará discapacitante para una persona con limitaciones funcionales, también para personas que se alejen del estereotipo de usuario (por ejemplo, una mujer embarazada o un niño)". Las tecnologías son clave para la promoción y prevención de la salud y la atención social, puesto que reducen las desigualdades y facilitando la inclusión social. Las tecnologías han de contribuir al desarrollo personal y a la igualdad de oportunidades, pero las dificultades de acceso y uso son, a menudo, uno de los factores de desigualdad entre los diferentes colectivos (Poveda, 2011).

Los productos y servicios diseñados y seleccionados bajo el conocimiento de la realidad de los usuarios, de sus capacidades y de sus necesidades facilitan la vida cotidiana a todas las personas, y de forma destacada, a las que tienen limitaciones funcionales.

Los productos de apoyo de la vida diaria van desde productos con alta carga tecnológica (por ejemplo, los sistemas domóticos) hasta productos más convencionales (mobiliario o calzado). La adecuación de todos ellos a los requisitos y necesidades de las personas mayores es importante para promover la autosuficiencia de las personas con dependencia.

Una manera de reducir la brecha entre las (TIC) y las necesidades asociadas al proceso de envejecer es la participación activa de las personas mayores en la investigación, para garantizar que las innovaciones tecnológicas se ajusten a sus necesidades. Para conocer la realidad y las necesidades de las personas dependientes con los productos es necesario contar desde el primer momento del diseño con el usuario real (persona mayor, profesional y cuidador no profesional). Esa participación activa del usuario es lo que diferencia al diseño centrado por el usuario del proceso de diseño tradicional. En los diseños orientados al usuario se generan herramientas para que los profesionales puedan captar los deseos y necesidades de los usuarios, buscar información, plantear problemas o 
BÚSQUEDA - Julio / Diciembre de 2015 - No. 15 (38 - 50)

proponer soluciones a la hora de planificar sus actividades.

Los objetivos principales de la tecnología en el entorno son la mejora o el mantenimiento de diversas funciones que hacen que las personas puedan tener una vida autónoma con suficiente movilidad dentro y fuera, que sirvan para el cuidado y la atención en el hogar, que den seguridad dentro y fuera del entorno familiar, que fomenten los estilos de vida saludables, que faciliten el desarrollo personal y la información y comunicación con otros, y que, a la vez, mantengan las relaciones sociales de la persona (Pinazo 2013; Pinazo, 2014; Pinazo y Poveda, 2015). Sepuede ver en la siguiente figura (Ver figura 1).

Algunos ejemplos que facilitan tener una vida autónoma con suficiente movilidad dentro y fuera del hogar son los productos destinados a evitar las caídas, los detectores de movimiento, la teleasistencia, las soluciones domóticas para apertura de puertas, climatización, etc. Con respecto al cuidado y atención en el hogar se han ido desarrollando diferentes soluciones basadas en la robótica: robots para estimulación cognitiva o con programas de ejercicio físico, robots de compañía para evitar la soledad o todos aquellos productos dirigidos al desarrollo personal y la información y comunicación con otros y mantenimiento de las relaciones sociales dirigidos a evitar el aislamiento (Donio-Bellegarde, Pinazo-Hernandis y Sánchez, 2013).

Por ejemplo, el proyecto SlforAGE (Social Innovation for Active and Healthy Ageing) (http:// www.siforage.eu) de prestación de servicios para las personas mayores con innovación centrada en la persona reúne a una amplia diversidad de agentes que representan a la sociedad civil, a usuarios finales (personas mayores), diseñadores de políticas públicas, think-tanks y expertos a nivel europeo e internacional, científicos, universidades, administraciones públicas y empresas con vistas a mejorar la competitividad de la Unión Europea en lo que se refiere a la promoción de la investigación y de productos innovadores que ayuden a vivir vidas más largas y saludables.

Figura 1. Objetivos de la tecnología en el entorno de las personas mayores

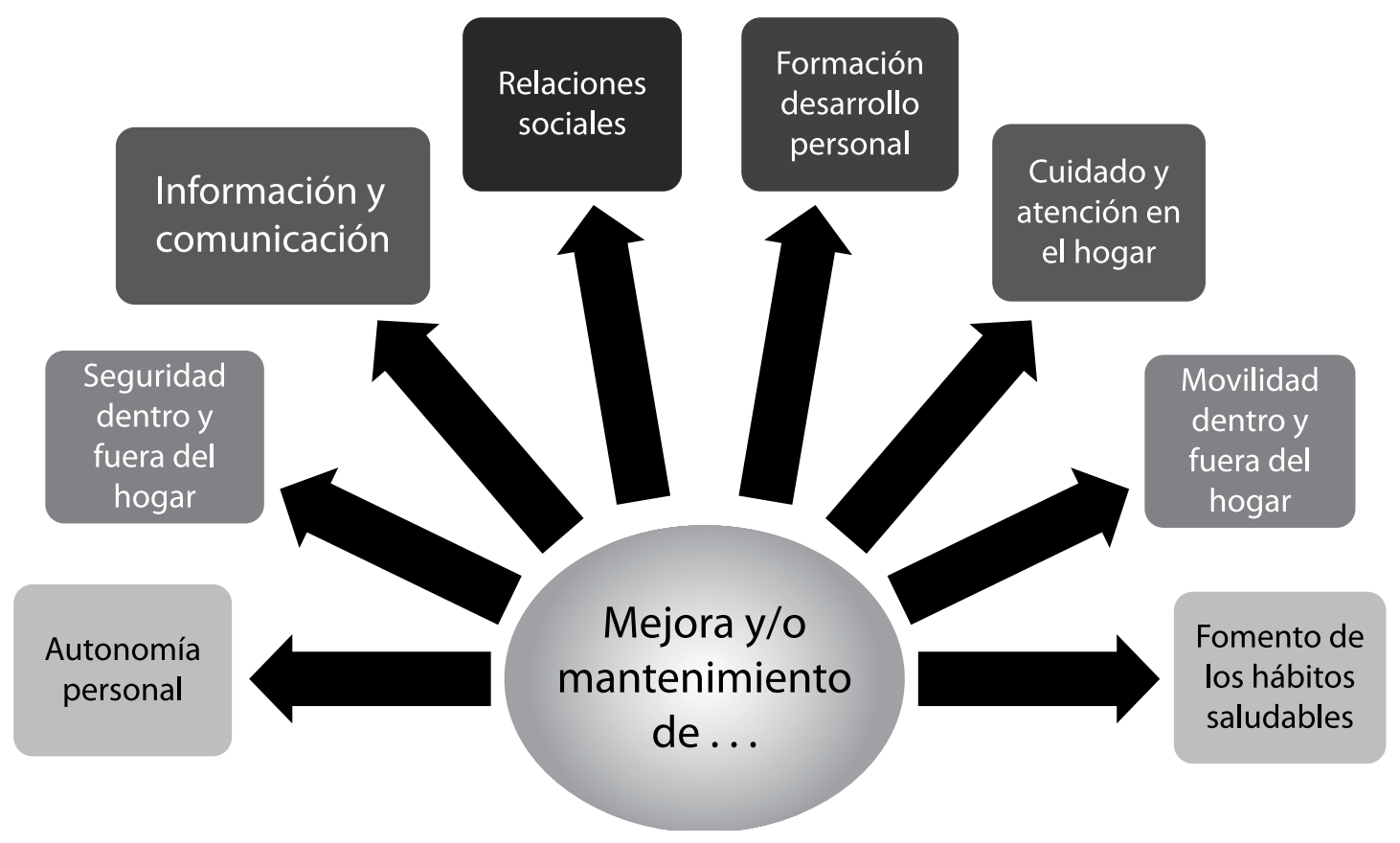

Fuente: Elaboración propia. 
Sacramento Pinazo-H., Rakel Poveda P., - Innovación para el envejecimiento activo en la unión europea

En el año 2008 se implantó el programa Europeo Ambient Assisted Living Joint Research and Development Programme (AAL), en el que participan diferentes países como: Austria, Bélgica, Chipre, Dinamarca, Eslovenia, España, Francia, Hungría, Irlanda, Luxemburgo, Países Bajos, Polonia, Portugal, Reino Unido, Rumanía, Suecia y Suiza. El Programa AAL está basado en el artículo 169 del Tratado de la Unión Europea. Aunque se inició con un duración prevista de 6 años (2008-2013), (http://www.aal-europe.eu/), en mayo de 2014 el Parlamento Europeo y el Consejo de la Unión Europea dieron continuidad al Programa AAL-2, (2014-2020) como programa de investigación y desarrollo, basado en los artículos 185 y 188 del Tratado de la Unión Europea y englobado dentro del Programa Horizonte 2020. El programa AAL está destinado a mejorar las condiciones de vida de las personas mayores, la calidad y eficacia de los sistemas de salud y bienestar social y fortalecer la base industrial de Europa a través del desarrollo y uso de TIC que respondan, desde un punto de vista multidisciplinar, a las necesidades de las personas mayores. La oportunidad de este programa se debe a la importancia creciente de las necesidades sociales y sanitarias derivadas del cambio demográfico con un progresivo envejecimiento de la población europea.

El término envejecimiento activo fue planteado por la OMS a finales de los años 90. Se define envejecimiento activo como "el proceso de optimización de las oportunidades de salud, seguridad y participación, con el fin de mejorar la calidad de vida a mediad que las personas envejecen (...) incluyendo a aquellas en situación de dependencia" (OMS, 2002: 79). "Las políticas y los programas de envejecimiento activo reconocen la necesidad de fomentar y equilibrar la responsabilidad personal (el autocuidado de la salud), los entornos adecuados para las personas mayores y la solidaridad intergeneracional. Las familias necesitan planificar su vejez, prepararse para ella y llevar a cabo esfuerzos personales para adoptar prácticas de salud positivas y personales en todas las etapas de la vida" (OMS, 2002: 81). Desde la OMS (2002) el envejecimiento activo se fundamenta en los derechos humanos de las personas mayores y en el reconocimiento de los Principios Universales de las Naciones Unidas para las personas de edad: independencia, autorrealización, participación, dignidad y cuidados.

Una vez conocidas y revisadas las diferentes convocatorias de AAL desde su implantación, los tipos de proyectos financiados y las áreas de desarrollo, los objetivos planteados en esta investigación fueron:

01. Analizar los proyectos de desarrollo de tecnologías de ayuda y apoyo a las personas mayores evaluados positivamente y realizados en los últimos años (2008-2015).

O2. Reflexionar sobre el futuro de las tecnologías en la promoción del envejecimiento activo.

\section{Metodología}

Con el fin de realizar una aproximación al estado del arte en materia de desarrollo tecnológico dirigido a personas mayores se seleccionó el programa AAL por ser la convocatoria europea más específica que financia la innovación en el ámbito de la gerontología y los cuidados mediante el desarrollo de TIC y que cuenta con la participación activa de las personas mayores, siendo un criterio, imprescindible para la elegibilidad de las propuesta, la participación usuarios finales (primarios, secundarios o terciarios). Se ha realizado un análisis documental de los proyectos disponibles en la página de AAL de las ocho convocatorias realizadas hasta 2015 y las web activas de los proyectos.

Como marco teórico de partida se ha basado en los principios del envejecimiento activo (OMS, 2002). El análisis documental realizado se centra en la comparación del programa con los principios del envejecimiento activo y la descripción de algunas de las soluciones desarrolladas. Tras una lectura en profundidad de los propósitos de cada convocatoria y mediante una rejilla de análisis, dos jueces revisaron el grado de 
BÚSQUEDA - Julio / Diciembre de 2015 - No. 15 (38 - 50)

cercanía entre los objetivos de la convocatoria AAL y el Paradigma del envejecimiento activo.

\section{Resultados}

Un total de ocho convocatorias han sido analizadas (http://www.aal-europe.eu/getinvolved/statistics/en; AAL_Catalogue_2014_ WEB.pdf). Las diferentes convocatorias se han dirigido a la mejora de la atención domiciliaria y enfermedades crónicas, mejora de la interacción social, participación e independencia en los servicios TIC, y mejora de la movilidad. El total de proyectos enviados a las convocatorias 1 a 6 ha sido de 646, y de ellos aparecen descritos y han recibido financiación, la primera fase de $\mathrm{AAL}$ (seis convocatorias desde 2008) 152 con una media de 8 partners por propuesta.

Las convocatorias, sus lemas y el número de proyectos han sido:

-Convocatoria 1, 2008, que lleva por lema: "Soluciones basadas en TIC para la prevención y gestión de enfermedades crónicas de las personas mayores". Dotada con 57,7 M€, 23 proyectos descritos y financiados de los 118 presentados.

-Convocatoria 2, 2009: "Soluciones TIC para la mejora de la interacción social de las personas mayores". Dotada con 60,9 M€, 32 proyectos descritos y financiados de los 104 presentados.

-Convocatoria 3, 2010: "Soluciones TIC para mejorar la independencia y la participación en la sociedad". Dotada con $54 \mathrm{M} €, 22$ proyectos descritos y financiados de los 91 presentados.

-Convocatoria 4, 2011: "Soluciones TIC para la mejora de la movilidad de las personas mayores". Dotada con 52,2 M€, 25 proyectos descritos y financiados de los 107 presentados.

-Convocatoria 5, 2012: "Soluciones TIC para la autogestión de las actividades de la vida diaria en los hogares de las personas mayores". Dotada con 49,39 M€, 29 proyectos descritos y financiados de los 151 presentados.
-Convocatoria 6, 2013: "Soluciones TIC para el apoyo de las actividades (participación y ocupación) en la vida de las personas mayores". Dotada con 42,34 M€, 21 proyectos descritos y financiados de los 75 presentados.

-Convocatoria 2014: "Cuidando para el futuro". Dotada con 33,8 M€.

-Convocatoria 2015: "Vida activa e independiente en el hogar". Dotada con 31,9 M€.

En el momento de cierre de este artículo se encuentra abierto el preanuncio de la Convocatoria 2016 (Ilamada a enviar propuestas), que no se analizarán aquí por falta de datos, y que lleva por lema: "Vivir bien con la demencia". La contribución de la TIC a las soluciones integradas para conseguir el bienestar de las personas que viven con demencia y sus comunidades ${ }^{3}$.

Los partners y los coordinadores de los diferentes proyectos enviados y los financiados pertenecen a los diversos países de la Unión Europea y Suiza, entre los que destaca una mayor presencia de proyectos españoles, italianos y alemanes, en ambos casos (como partners y como coordinadores) y desde pequeñas y medianas empresas, universidades, centros de investigaciones y asociaciones de usuarios finales (personas mayores). Las diferentes convocatorias hasta la fecha de inicio de esta investigación se han dirigido a aspectos como la mejora de la atención domiciliaria y la atención a las enfermedades crónicas, la mejora de la interacción social, la participación y la vida independiente, y la mejora de la movilidad. $\mathrm{Si}$ se sigue el Paradigma del envejecimiento activo, los proyectos se han dirigido a la mejora de la participación, salud y seguridad. El fin general que ha guiado todas y cada una de las convocatorias ha sido aumentar la autonomía, la participación en la vida social, las habilidades y el empleo en las personas mayores.

Se quiso analizar en profundidad cada una de las convocatorias siguiendo el Paradigma

\footnotetext{
${ }^{3}$ Ver más en: http://www.aal-europe.eu/pre-announcementof-the-aal-call-challenge-2016/\#sthash.fRiq3dRd.dpuf
} 
Sacramento Pinazo-H., Rakel Poveda P., - Innovación para el envejecimiento activo en la unión europea

del envejecimiento activo. En la figura 2 que se muestra a continuación (ver figura 2) se puede ver la relación de las convocatorias y los principios del envejecimiento activo.

Se destaca en primer lugar que las diferentes convocatorias se han focalizado hacia uno de los aspectos del envejecimiento o a veces en varios de ellos a la vez. Son muchas las convocatorias centradas en la mejora o mantenimiento de la salud y capacidad funcional, por ejemplo, las Convocatorias 1 y 7 . La primera de las convocatorias de AAL iba dirigida a proyectos que proporcionasen soluciones innovadoras y con una aproximación holística para la prevención, manejo y servicios de apoyo basados en las TIC para personas mayores con factores de riesgo identificados y/o enfermedades crónicas (diabetes, enfermedades cardiovasculares, esclerosis múltiple o enfermedad de Alzheimer. Por su parte, la Convocatoria 7, "Cuidando para el futuro" innova en cómo las soluciones basadas en las TIC podrían atender a las crecientes demandas, incrementar y facilitar el suministro de cuidados formales e informales a las personas mayores, reducir las demandas de cuidados a través de la prevención y el autocuidado, y apoyar el cambio hacia un mejor cuidado tanto en el domicilio como en la comunidad.

Las Convocatorias 2, 3, y 6 han ido dirigidas fundamentalmente a aspectos relacionados con la participación social, aunque es sabida la relación entre participación/inclusión social y bienestar, por tanto también se podría decir que se trabaja el otro pilar del envejecimiento activo: la salud. La Convocatoria 2 financió proyectos TIC dirigidos a ayudar a las personas a estar activas, felices y conectadas socialmente y que contribuyesen a mejorar/mantener su salud, calidad de vida e inclusión social. La Convocatoria 3 se centró en proyectos que ayudasen a preservar la independencia y la dignidad en todos los aspectos de la vida, para posibilitar que los mayores tomasen una parte activa en la sociedad, por lo que contribuye a estimular y apoyar las capacidades requeridas para dicha participación (desde la movilidad hasta aspectos más físicos o cognitivos). La Convocatoria 6 se dirigió a proyectos cuyo fin fuese promover la actividad de los adultos mayores porque la ocupación es crucial para dotarlos de un

Figura 2. Convocatorias AAL y relación con los principios del envejecimiento activo

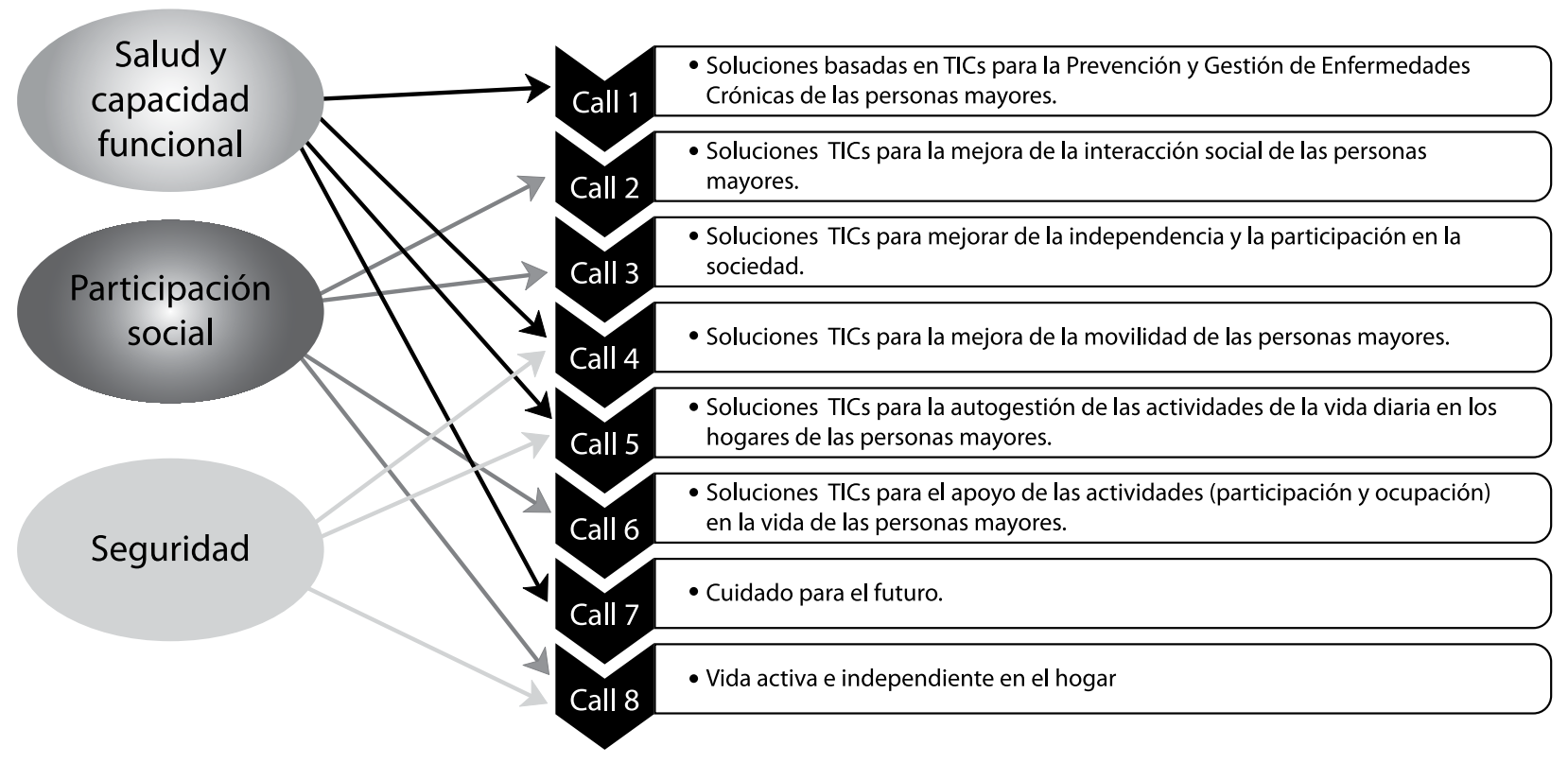

Fuente: Elaboración propia a partir de los datos del catálogo AAL 2014: proyectos financiados. 
BÚSQUEDA - Julio / Diciembre de 2015 - No. 15 (38 - 50)

propósito en la vida. Algunos de sus objetivos son: diseñar productos que ayudasen a preservar las capacidades físicas y cognitivas de quienes ya se hubiesen jubilado y ayudarlos en sus puestos de trabajo, adaptando o rediseñándolo, formulando nuevos modos de aprendizaje en el ámbito laboral. Sabiendo que el año 2012 fue el Año europeo del envejecimiento activo y la solidaridad intergeneracional, esta convocatoria también iba dirigida a proyectos que se centrasen en posibilitar la transferencia intergeneracional de conocimientos para aprovechar el caudal de experiencias de los más mayores.

La Convocatoria 5, se centró en proyectos basados en las TIC que mostrasen el potencial de activar y apoyar en las actividades de la vida diaria de las personas mayores y apoyar a los cuidadores para proporcionar asistencia. Esta convocatoria iba dirigida a aspectos relacionados con la seguridad y la salud y capacidad funcional.

La Convocatoria 8 se focalizó en aspectos relacionados con la seguridad, pero también con la participación social. La idea fundamental es ayudar a las personas a vivir de manera independiente durante el mayor tiempo posible en sus hogares proporcionándoles la menor ayuda profesional necesaria y siempre desde la posibilidad de elegir y tomar sus propias decisiones; además, ayudar a vivir vidas lo más integradas posibles en el ambiente comunitaria en la perspectiva de aumentar la participación social.

La Convocatoria 4 se centró en las ayuda que pueden proporcionar las TIC para la movilidad de las personas mayores, conociendo la bidireccionalidad entre salud y movilidad, salud, seguridad y participación. El mantenimiento de la movilidad y la orientación es un factor clave en el envejecimiento activo, que permite a las personas mayores tener vidas dinámicas e independientes, además de ser un factor fundamental para la participación e inclusión social. Las personas que tienen movilidad autónoma o cuentan con los suficientes recursos de apoyo para que las dificultades en la movilidad sean minimizadas, pueden realizar de manera autónoma las AVD, son capaces de mantener su red social, y elegir qué hacer en diferentes momentos de su vida diaria. La movilidad retrasa la discapacidad y pospone la fragilidad. La relación entre falta de movilidad, soledad y depresión ha sido manifestada por diversas investigaciones; otros estudios han mostrado la relación entre ejercicio físico y salud física, cognitiva y emocional.

Se aspira a destacar algunos ejemplos de proyectos realizados con participación de socios españoles en las diferentes convocatorias, que aparecen en el catálogo de AAL como:

Con respecto a la Convocatoria 1 (2008), denominada "Soluciones basadas en TIC para la prevención y gestión de enfermedades crónicas de las personas mayores", se destaca el proyecto AMICA (Autonomy, Motivation e Individual SelfManagement for COPD Patients), coordinado por la Universidad de Cádiz y dirigido a personas con enfermedad pulmonar obstructiva crónica (EPOC). El objetivo es la mejora de la gestión y la atención médica a estos pacientes, y para ello se desarrolló una plataforma que proporciona a los pacientes, cuidadores y médicos, herramientas que permitiesen la prestación de la atención en el domicilio. Gracias a su diseño ergonómico podía ser usada por el mismo paciente de manera autónoma.

En lo referente a la Convocatoria 2 (2009), denominada "Soluciones TICs para la mejora de la interacción social de las personas mayores", destacamos el Proyecto AWARE (Ageing Workforce towards an Active Retirement), que fue coordinado por el Instituto de Biomecánica de Valencia (IBV) y tuvo como objetivo proporcionar a las personas mayores, tanto trabajadoras como jubiladas, habilidades en el uso de las TIC y promover el envejecimiento activo, a la vez que ayudaba la preparación a la jubilación a las personas que se encontraban en ese proceso. Otro proyecto interesante a destacar es el Proyecto CVN cuyo objetivo es conectar, a través de redes de vídeo, grupos de mayores con intereses comunes y capacidades y estilos de vida similares. El Proyecto SilverGame ha estado dirigido al desarrollo de aplicaciones multimedia para la creación de juegos que favorezcan la 
Sacramento Pinazo-H., Rakel Poveda P., - Innovación para el envejecimiento activo en la unión europea

interacción social y la transferencia de estas interacciones virtuales a la vida real.

De la Convocatoria 3 (2010), que llevaba por título "Soluciones TIC para mejorar la independencia y la participación en la sociedad", vale la pena destacar el Proyecto BANK4ELDER (Innovate Ways of Banking Designed for and by the Elderly), coordinado por Vector y con la participación del Instituto de Biomecánica de Valencia y Rutis (Universidad de mayores). Este proyecto ha permitido diseñar y desarrollar interfaces amigables en los cajeros automáticos, la banca por internet, la televisión y el teléfono móvil y así ayudar a un acceso más sencillo a los servicios bancarios.

En la Convocatoria 4 (2011), "Soluciones TIC para la mejora de la movilidad de las personas mayores" vale la pena destacar el Proyecto ALICE (Assistance for Better Mobility and Improved Cognition of Elderly Blind and Visually Impaired), que contó con la participación de la empresa española Information and Image management Systems, S.A, Tenía como objetivo el desarrollo de un dispositivo con habilidades de navegación y cognitivas para personas mayores ciegas.

Con respecto a la Convocatoria 5 (2012) "Soluciones para la autogestión de las actividades de la vida diaria en los hogares de las personas mayores mediante TIC" queremos destacar el Proyecto WETAKECARE, coordinado por el IBV y cuyo objetivo es capacitar en el cuidado a la persona mayor y el cuidador no profesional de una manera colaborativa, con el fin de promover la vida independiente de las personas mayores mediante el uso de juegos y material formativo alojados en una plataforma usable y adaptable a las necesidades de los usuarios finales.

En lo referente a la Convocatoria 6, "Soluciones TIC para el apoyo de las actividades (participación y ocupación) en la vida de los adultos mayores", destacar el Proyecto COGNIWIN (Cognitive Support for Older Adults at Work) cuyo objetivo es adaptar las tareas de las personas mayores a su capacidad cognitiva.

Finalmente, la Convocatoria 7 denominada "Cuidando para el futuro", tiene como objetivo financiar el desarrollo y la validación de soluciones TIC para la creación de modelos sostenibles de atención para las personas mayores.

La Convocatoria actual, la 8 (2015) que se ha denominado "Vida activa e independiente en el hogar", se plantea como objetivo dar respuesta, mediante proyectos innovadores, transnacionales y multidisciplinares, a una mayor independencia en su hogar y una mayor autodeterminación de las personas mayores mediante el uso de TIC.

Tal y como indica el programa AAL la participación de los usuarios finales en los proyectos de investigación tiene como principal beneficio el desarrollo de tecnología centrada en la persona que facilite estrategias de mercado desde el conocimiento de las necesidades reales de los usuarios. El programa AAL define y establece los beneficiarios de los servicios o productos AAL como se muestra en la siguiente tabla (ver tabla 2):

Tabla 1. Usuarios y beneficiarios de los servicios o productos AAL

\begin{tabular}{lll}
\hline \multicolumn{1}{c}{ Usuario } & Tipo & Beneficio esperado \\
\hline $\begin{array}{l}\text { Primario o beneficiario final } \\
\begin{array}{l}\text { Secundarios } \\
\text { Terciarios }\end{array}\end{array}$ & Persona mayor & Mejora de la calidad de vida \\
& $\begin{array}{l}\text { Personas organizaciones directamente } \\
\text { en contacto con el usuario primario } \\
\text { (cuidadores, amigos...) }\end{array}$ & Reducción de las necesidades de cuidado \\
& $\begin{array}{l}\text { Instituciones y organizaciones públicas } \\
\text { o privadas }\end{array}$ & $\begin{array}{l}\text { Mayor eficiencia y eficacia en la prestación } \\
\text { de servicios que se traducen en ahorro de } \\
\text { gastos o mantenimiento de estos. }\end{array}$ \\
\hline
\end{tabular}


BÚSQUEDA - Julio / Diciembre de 2015 - No. 15 (38 - 50)

Algunas de las entidades participantes como usuarios en los proyectos han sido: a. En los proyectos cuyo objetivo principal es la mejora de la salud han sido instituciones y organizaciones públicas como hospitales, fundaciones o servicios asistenciales (por ejemplo: Badalona Serveis Assistencials o la Fundación Hospital Comarcal). b. Los proyectos dirigidos a la participación destacan las asociaciones de usuario (por ejemplo: UDP o VASOS) y a seguridad (prestadores de servicios para personas mayores).

La mayoría de los proyectos, cuyos resultados son prototipos funcionales que pueden ser puestos en el mercado con desarrollos definitivos posteriores al proyecto, tiene en cuenta a población mayor de 50 años con el objetivo de incluir a las nuevas generaciones de personas mayores, puesto que la aceptación de la tecnología como herramientas para la mejora de la calidad se prevé más alta que en la actualidad.

\section{Conclusiones}

El reto de las sociedades envejecidas exige un cambio de modelo de atención a la vez que una real incorporación de las tecnologías para conseguir dar respuesta a las diferentes demandas, necesidades y situaciones de los ciudadanos mayores y les permita alcanzar un envejecimiento activo.

Las tecnologías con diseños adecuados a la realidad de todos los usuarios son elementos fundamentales para que todas las personas puedan desarrollar sus derechos de la manera más independiente posible. Entre las funciones principales se destacan: el apoyo en la realización de las actividades de la vida diaria, la permanencia en el propio hogar, una mejor atención a las personas en situación de dependencia, la reducción del coste de la atención, la mejora de la inclusión social, la reducción de los problemas de aislamiento y soledad, y la prevención de situaciones de dependencia (por ejemplo, la reducción de probabilidad de caídas). Teniendo en cuenta que problemas como este último, son de gran importancia dado que las caídas son la segunda causa mundial de muerte por lesiones accidentales o no intencionales, y que los mayores de 65 años son quienes sufren más este tipo de accidentes, es necesaria la intervención desde todas las esferas posibles de la atención.

Las estrategias preventivas deben hacer hincapié en la educación, la capacitación, la creación de entornos más seguros, la priorización de la investigación relacionada con la prevención de la dependencia y el fomento del envejecimiento activo.

El reconocimiento del valor de las TIC es muy grande desde sectores como los centros de investigación, las asociaciones y fundaciones de personas mayores y sus familiares, los profesionales de la intervención, los proveedores y desarrolladores de productos y servicios y las propias administraciones públicas. Pero desde hace varias décadas se demanda la implementación real de las normas técnicas y legislativas de accesibilidad universal y diseño para todos, una mayor financiación de actividades de $\mathrm{I}+\mathrm{D}+\mathrm{i}$ que asegure el desarrollo e implementación en el mercado de estas tecnologías de forma asequible, los procesos de evaluación continua de la calidad incorporando la medición de la satisfacción de los usuarios en estos procesos, la mejora de los procesos de provisión y análisis de coste-benéfico, el desarrollo de un sistema de información sobre el sistema de provisión, la promoción del uso de dispositivos técnicos, un desarrollo legislativo, nuevos materiales formativos, la concienciación de los usuarios sobre las capacidades, mayores recursos dedicados a la información, asesoramiento en el uso y formación de los profesionales y apoyo al desarrollo e implementación de servicios desarrollados bajo el paraguas de la innovación social.

El programa AAL se enfoca principalmente al desarrollo de TIC que permitan a la persona mayor permanecer en su hogar el mayor tiempo posible de forma autónoma e independiente, desarrollar soluciones que reduzcan el coste de la atención y la brecha digital, todo ello contando 
Sacramento Pinazo-H., Rakel Poveda P., - Innovación para el envejecimiento activo en la unión europea

con la participación activa de los usuarios con el fin de encontrar las soluciones más adecuadas a la realidad de las personas mayores del siglo XXI. Fundamentado en el Paradigma del envejecimiento activo y los Principios Universales de las Naciones Unidas para las personas de edad (independencia, autorrealización, participación, dignidad y cuidados), en líneas generales los proyectos han ido dirigidos a la promoción de la salud y la actividad autónoma y a la monitorización de la salud, en áreas como la inclusión social (apoyo, fomento de las relaciones sociales), a la prevención (de fragilidad, de demencia, de potenciales enfermedades), a la supervisión (adherencia al tratamiento médicofarmacológico, enfermedades crónicas, signos vitales) a la detección (de riesgos, de problemas de salud), al apoyo (rehabilitación físico-funcional, acompañamiento emocional y psicológico a distancia). Para ello, los diferentes proyectos han desarrollado técnicas como las interfaces de uso inteligente, las aplicaciones fáciles de usar para todos y tecnologías como los sensores, el GPS, el reconocimiento de imágenes o voz por video. Los avances en la comunicación persona-ordenador sobre todo con la tecnología $4 \mathrm{G}$ y el desarrollo de los móviles (teléfonos inteligentes o smartphones) han abierto un sinfín de posibilidades.

En la actualidad, diferentes áreas siguen desarrollándose para el fomento del envejecimiento activo y aún queda camino por andar. La mejora de la seguridad en los domicilios y fuera de ellos pero también en las instituciones (tecnología para la automatización del hogar, sistemas de control, alarmas y seguimiento mediante $\mathrm{TIC}$ ); proyectos y programas que se centren en el mantenimiento y mejora de la salud (soluciones integrales para la atención a la dependencia, mejora de la autonomía mediante productos de apoyo y sistema robóticos, aplicaciones para el telecuidado, telemedicina y telerehabilitación); ideas innovadoras que faciliten la participación de las personas mayores en las sociedades en las que viven (desarrollo y mejora de redes sociales, TIC, aplicaciones para la mejora del ocio, mejora de la accesibilidad, aceptabilidad y usabilidad de las tecnologías).
La población europea envejece con el consiguiente aumento de los costes de asistencia sanitaria y social. Las TIC pueden ayudar a hacer sostenibles los sistemas de asistencia sanitaria y social, reduciendo sus costes y aumentando su eficiencia, mejorando la calidad de vida de las personas mayores en el trabajo, en la comunidad y en el hogar. Las diferentes convocatorias de AAL han intentado cubrir los principales retos del envejecimiento mediante el desarrollo de TIC amigables y sistemas para el cuidado y la atención de las personas mayores en el marco del envejecimiento activo. Pero este avance tecnológico sirve de poco si los profesionales sociosanitarios no son conocedores de las nuevas propuestas. Se considera de sumo interés que los profesionales sociosanitarios puedan conocer el avance y la innovación en las TIC y este artículo pretende aportar una reflexión sobre el tema.

\section{Referencias bibliográficas}

AAL (2015). Active Assisted Living Programme ICT for ageing well. Recuperado el 2 de octubre de 2015 de http://www.aal-europe. eu/wp-content/uploads/2015/02/AAL_ Catalogue_2014_WEB.pdf

AAL (2015). Overall statistics on Calls. Recuperado el 2 de octubre de 2015 de http://www.aaleurope.eu/wp-content/uploads/2015/05/ Overall-statistics-on-calls.pdf

CEAPAT (2015). Tecnología y personas mayores. Madrid: CEAPAT-IMSERSO.

Colombo, F. et al (2011), Help Wanted? Providing and Paying for Long - Term Care, OECD Publishing. Recuperado el 10 de noviembre de 2015 de http:// www.oecd.org/health/ longtermcare/helpwanted

Donio- Bellegarde, M., Pinazo-Hernandis, S. y Sanchez, F. (2013), As TIC como instrumento para reduzir a solidão e o isolamento social na velhice: Uma revisão bibliográfica. VII Congreso Norte-Nordeste de la Sociedad Brasileña de Geriatria y Gerontologia, Fortaleza, Brasil, Desafios 
BÚSQUEDA - Julio / Diciembre de 2015 - No. 15 (38 - 50)

do Envelhecimento: Atividade, Autonomia e Qualidade de Vida.

Echevarría, J. (2008). El manual de Oslo y la innovación social. Arbor, 184(732), 609618.

European Commission (2003). Feasibility Study, comparable statistics in the area of care of dependent adults in the European Union. Final report.

European Commission (2007). Healthy ageing: keystone for a sustainable, Luxembourg: European Commission. Recuperado 4 de octubre de 2015, a partir de http:// ec.europa.eu/health/ph_information/ indicators/docs/healthy_ageing_en.pdf

Fernández J., Parapar, C. y Ruiz, M. (2010). envejecimiento de la población. Lychnos. Cuadernos de la Fundación CSIC, 4-12, 2.

HelpAge International (2015). Global Age Watch Index: Insight report, summary and methodology. Recuperado 22 de septiembre de 2015, a partir de http://www. helpage.org/global-agewatch/reports/ global-agewatch-index-2015-insightreport-summary-and-methodology/

ILC-Br (2015). Active Ageing: A policy framework in response to the longevity revolution. Brasil: International Longevity Centre Brazil.

IMSERSO (2012). Las personas mayores en España. Madrid: IMSERSO.

OMS (2002). Envejecimiento activo: un marco político. Revista Española de Geriatría y Gerontología, 37 (S2), 74-105.

OMS (2010). Global Health and Aging. National Institute on Aging.

OMS (2015). Diez datos sobre el envejecimiento y la salud. Recuperado 4 de octubre de 2015, a partir de http://www.who.int/ features/factfiles/ageing/es/
Pinazo-Hernandis, S. (2013). Tecnologías emergentes no cuidado do idoso. Conferencia inaugural en VII Congreso Norte-Nordeste de la Sociedad Brasileña de Geriatría y Gerontología, Fortaleza, Brasil, Desafios do Envelhecimento: atividade, autonomia e qualidade de vida.

Pinazo-Hernandis, S. (2014). Tecnologías emergentes no cuidado do idoso. Conferencia en XIX Congresso Brasileiro de Geriatria y Gerontologia, Belem, Brasil.

Pinazo-Hernandis, S. (2015): Avances en Gerontología. Desafíos ante una Sociedad que envejece. Conferencia en V Congreso Internacional de Gerontología: Avances de la Gerontología: Desafíos ante una sociedad que envejece. San José, Costa Rica.

Pinazo-Hernandis, S. y Poveda, R. (2015). Tecnologías de ayuda para la mejora en la calidad de vida de los adultos mayores. Conferencia en V Congreso Internacional de Gerontología: Avances de la Gerontología: Desafíos ante una sociedad que envejece. San José, Costa Rica.

Poveda, R. (2011). Diseño de bienes y servicios centrados en las personas mayores. En II Foro sobre innovación, economía y calidad de vida (pp. 103-110). Valencia: Instituto de Biomecánica de Valencia.

Poveda, R., Belda, J., Barberà, R., Cort, JM., Prat, JM, Matey, F, Soler, C y Dejoz, R. (2005). Facilitación de la vida cotidiana mediante el diseño y la selección adecuada de tecnologías de apoyo para personas mayores con dependencia funcional. Revista Multidisciplinar de Gerontología, 15 (3), 155-164.

SlforAge consortium: Providing innovative solutions for an active and healthy ageing. (2015). Recuperado 4 de octubre de 2015, a partir de http://www.siforage.eu/index. php 